\title{
Análise comparativa entre estimativas do modelo SWAN e medições de agitação marítima efectuadas na Praia da Amoreira, Portugal *,***
}

\author{
Comparative analysis between SWAN's numerical estimates and sea wave \\ measurements made at the Amoreira's Beach, Portugal
}

\author{
Rui Capitão ${ }^{\circledR, 1}$, Conceição Fortes ${ }^{1}$
}

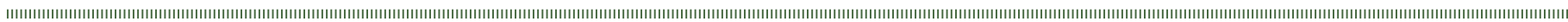

\section{RESUMO}

Neste trabalho, analisa-se o desempenho do modelo numérico SWAN (Booij et al., 1999) na caracterização da agitação marítima na zona costeira da praia da Amoreira, em Aljezur, Algarve. Para este efeito, efectua-se uma análise comparativa entre as mediçóes de agitação marítima realizadas na Praia da Amoreira e as estimativas produzidas por esse modelo. Assim, partindo dos resultados do modelo regional de previsão da agitação marítima, WAVEWATCH III, Tolman (1999, 2002), para os dias em que foram realizadas as medições in-situ, efectuaram-se cálculos com o modelo SWAN, obtendo-se nos locais de medição valores de altura significativa, período de pico e período médio. A comparação das simulaçóes numéricas com os dados medidos permitiu avaliar o desempenho do modelo numérico e reforçar a confiança que já existia neste modelo na sua utilização em estudos de morfodinâmica e dinâmica costeira.

Palavras-chave: Ribeira de Aljezur (Portugal), Mediçôes in-situ, Modelação numérica, SWAN

\section{ABSTRACT}

The understanding of hydrodynamics, morphodynamics and fecal contamination of intermittent coastalstreams encompasses an interdisciplinary study that integrates three different methodologies, although complementary: acquisition of field data, laboratory investigation and numerical modeling. The results of this methodology in the study area, the Ribeira de Aljezur, will enable the assessment of the relative importance of various forcings on the morphology of the system and its impact on the water quality of generic coastal streams.

In this work a sea wave characterization is made on the intermittent-type Aljezur stream, Algarve, Portugal, to understand the hydrodynamics, morphodynamics and fecal contamination effects, as well as its impact both on the water quality and on the evolution of the bottom and of the water exchanges in the region under study. Thus, in-situ measurements of sea waves were made using pressure transducers at points near the mouth of the river and on the Amoreira's Beach, at the mouth of Aljezur stream. Numerical modeling was also employed to obtain a spatial characterization of the study area, through the use of SWAN numerical model (Booij et al., 1999). This model enables sea-wave generation, propagation and dissipation based on the wave action balance equation and is used to propagate sea waves from offshore up to the shoreline, taking into account the major physical processes of wave refraction, diffraction and shoaling due to bottom depth variation and to the presence of 
currents. It also includes wind induced wave growth, wave breaking due to bottom variation and to whitecapping, energy dissipation by bottom friction, wave blocking and reflection by opposing currents as well as wave transmission.

In order to evaluate the performance of this numerical model to characterize the sea waves at this beach, and to get even more confidence on this well-known model, currently used in many studies of coastal morphodynamics and long-term dynamics, a comparative analysis between measurements of sea waves made at Amoreira beach and the estimates produced by this model was performed. Thus, based on the results of the regional model prediction of sea waves, WAVEWATCH III, Tolman (1999, 2002), and for the days where in-situ measurements were taken, calculations were made with the SWAN model, resulting in significant wave heights, peak periods and mean periods for an area adjacent to the Amoreira beach, where those measurements were taken. A comparison of numerical simulations with the measured data allowed the evaluation of the behavior of the numerical model to simulate the propagation of sea waves on the beach.

In this work, the measurement campaigns, the analysis of the data obtained at the measuring campaigns and the application made with SWAN model to characterize the wave field generated by the wind at the study area were described and a comparison of numerical simulations with the in-situ measured data allowed the evaluation of the performance of the SWAN model for this location, guaranteeing even more confidence in its use in studies of morphodynamics and coastal dynamics.

Keywords: Ribeira de Aljezur (Portugal), in-situ measurements, Numerical modeling, SWAN

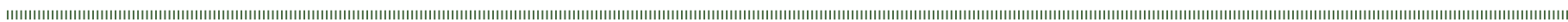

\section{INTRODUÇÁO}

A ribeira de Aljezur situa-se no extremo sul da praia da Amoreira, localizada no Parque Natural do Sudoeste Alentejano e Costa Vicentina (Figura 1). O local em estudo, onde desagua a Ribeira de Aljezur, apresenta-se como um sistema de pequenas dimensōes mas de elevada complexidade, com elevada variabilidade morfológica na zona da embocadura, com interrupções ocasionais da sua ligação ao mar, as quais são o resultado da acção conjunta da agitação marítima, correntes de maré e caudais fluviais intermitentes.

Para caracterizar a hidrodinâmica do sistema e o seu impacto na evolução de fundos e nas trocas de água na regiáo em estudo, é necessário avaliar a agitação marítima local. No presente trabalho, essa avaliaçáo é efectuada recorrendo a medições in-situ e a modelação numérica.

Foram realizadas quatro campanhas de campo de curta duração (1 dia), de 2008 a 2010, com mediçôes insitu da agitação marítima, utilizando sensores de pressão posicionados em locais junto à embocadura da ribeira de Aljezur e na zona de espraiamento da praia da Amoreira. Estas mediçóes constituem um conjunto de dados valioso para validação/calibração de modelos numéricos de propagação de ondas e permitem tirar algumas conclusóes sobre as características principais da agitação verificadas naqueles dias das campanhas. No entanto, dado o pequeno número de medições, e a sua curta duração, não é possível, com base nestas mediçóes, definir um regime de agitação marítima nem avaliar os efeitos a longo-prazo da agitação na morfologia do sistema em estudo.

Torna-se, por isso, interessante o recurso à modelação numérica, o que permite obter uma caracterização espacial e temporal da agitação marítima na zona em estudo.

Um dos modelos mais utilizados para este objectivo é o
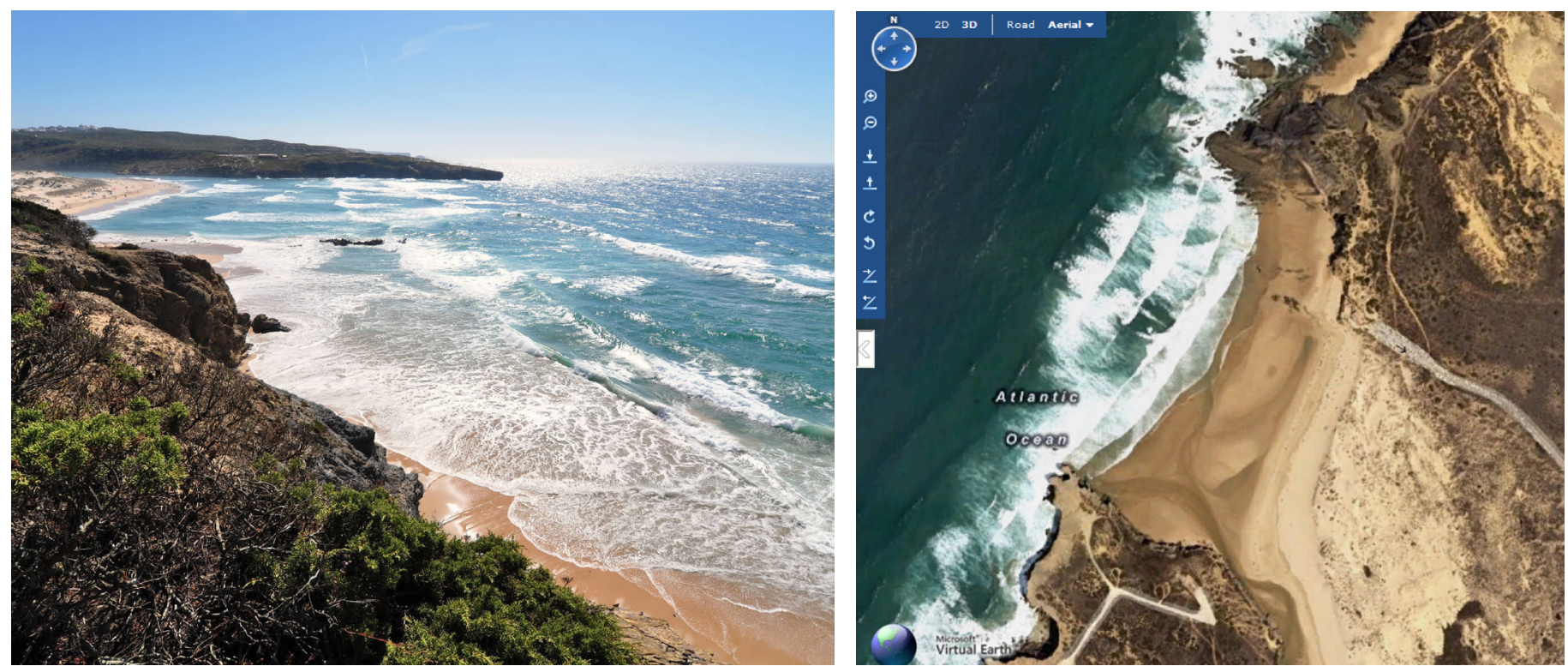

Figura 1. Localização da área de estudo: Praia da Amoreira (Virtual Earth@, 2010).

Figure 1. Study area location: Amoreira's beach (Virtual Earth (C, 2010). 
SWAN (Simulation WAves Nearshore), modelo espectral de alta resolução que simula e descreve a propagação e dissipação de ondas em zonas costeiras, (Booij et al., 1999). A partir do conhecimento das condiçóes de fronteira, batimetria, campos de vento e correntes, o modelo calcula a evoluçáo do espectro direccional, considerando todos os processos relevantes para uma descrição adequada da agitaçáo marítima em águas costeiras como a refracção devido à variação da profundidade, a dissipação por atrito no fundo, as interacçôes náo lineares ressonantes e a rebentação.

A maioria das aplicaçóes deste modelo à costa portuguesa tem sido realizada no âmbito de sistemas operacionais de previsão da agitação marítima, Rusu et al. (2008a, b) e Silva et al. (2009), por exemplo.

Existem vários trabalhos de análise de desempenho do modelo SWAN quando aplicados à costa Portuguesa, tais como as que resultaram da sua aplicação à zona marítima de Pinheiro da Cruz, Pires-Silva et al. (2002) ou Teles et al. (2008), ou à zona confinada da praia do Alfeite, no estuário do Tejo, Santos et al. (2007), Capitáo et al. (2009) e Rusu et al. $(2009,2011)$, em que foram efectuadas mediçôes de campo, ou a situaçóes em que se dispunha de dados de bóia-ondógrafo, tais como a zona marítima dos portos de Sines, Leixões e Faro, Capitão et al. (2006), Rusu et al. (2005a, 2005b, 2008b) e Silva et al. (2009), ou a zonas do Arquipélago da Madeira, Rusu et al. (2008a), e do Arquipélago dos Açores, na zona marítima do porto da praia da Vitória, Guilherme et al. (2009) e Santos et al. (2009). Em todos estes trabalhos, o estabelecimento das condiçóes de aplicação do modelo, bem como a calibração dos seus parâmetros, está fortemente condicionada pelo local de estudo, e, consequentemente, a precisão do modelo depende dessas condições e desses parâmetros.

Nesse sentido, e de modo a avaliar o desempenho deste modelo numérico na caracterização da agitação marítima na praia da Amoreira, e, assim, reforçar a confiança que existe actualmente na sua utilização generalizada em estudos de morfodinâmica e dinâmica costeira a longo prazo, efectuouse, neste trabalho, uma análise comparativa entre as mediçóes de agitação marítima locais e as estimativas produzidas por esse modelo.

Assim, partindo dos resultados do modelo regional de previsão da agitação marítima, WAVEWATCH III, Tolman $(1999,2002)$, para os dias em que foram efectuadas medições in-situ, efectuaram-se cálculos com o modelo SWAN, obtendo-se valores de altura significativa, período de pico e período médio na zona marítima adjacente à praia da Amoreira, nos exactos pontos onde foram efectuadas as referidas mediçóes in-situ. A comparação das simulaçóes numéricas com os dados medidos permite avaliar o comportamento do modelo numérico na simulação da propagação da agitação marítima na praia da Amoreira.

Neste artigo, descrevem-se as mediçóes efectuadas na praia da Amoreira, e as respectivas análises temporal e espectral, apresenta-se o modelo SWAN, descreve-se a aplicação efectuada com este modelo para a zona em estudo e discutem-se as comparaçóes efectuadas entre os valores estimados com o modelo e os dados medidos.

\section{MEDIÇÓES IN-SITU E TRATAMENTO DOS DADOS RECOLHIDOS}

Neste trabalho, a aquisição de dados de agitação marítima foi efectivada através da utilização de sensores de pressão colocados quer junto à embocadura da ribeira de Aljezur quer ao longo da praia da Amoreira. Consoante as campanhas, foram considerados vários locais de recolha de dados, com diferentes intervalos de amostragem e usando diferentes equipamentos de medida. Esta variabilidade de locais de medição e de equipamentos instalados deveu-se ao facto de se ter aproveitado este trabalho para afinar métodos de medição e para verificar a adequabilidade dos equipamentos à tarefa proposta.

Os instrumentos de medição utilizados na $1^{a}$ campanha (Campanha 1) foram sensores de pressão Honeywell, com cabo. Nas restantes campanhas (Campanhas 2, 3 e 4), para além destes sensores, utilizou-se também um sensor de pressão autónomo Infinity_WH. Ambos os sensores têm as características apresentadas na Tabela 1.

Tabela 1. Características dos sensores de pressão.

Table 1. Pressure transducer characteristics.

\begin{tabular}{l|l|l}
\hline Sensor & Honeywell & Infinity_WH \\
\hline Tipo de sensor & Piezo-eléctrico & Semi-condutor \\
\hline $\begin{array}{l}\text { Gama de mediçáo } \\
(\mathbf{m})\end{array}$ & $0-10$ & $0-25$ \\
\hline $\begin{array}{l}\text { Frequência de } \\
\text { aquisiçáo (Hz) }\end{array}$ & 25 & 10 \\
\hline Modo de aquisiçáo & $\begin{array}{l}\text { Cabo co-axial } \\
+ \text { PC. }\end{array}$ & $\begin{array}{l}\text { "Logger" mini-SD, com } \\
\text { conversor A/D 16-bit, } \\
\text { modos contínuo \& } \\
\text { "burst". }\end{array}$ \\
\hline
\end{tabular}

As características das 4 campanhas de campo em que foram realizadas aquisiçóes de dados de agitação marítima pelo LNEC podem ser consultadas na Tabela 2 e na Figura 2.

Os instrumentos operaram à cota da baixa-mar (relativamente à base do sensor), isto é, foram colocados em baixa-mar, com o sensor a tocar na superfície (nível médio) da água.

$\mathrm{O}$ posicionamento destes instrumentos variou da primeira campanha para as restantes, devido a um melhor conhecimento da hidrodinâmica da zona em estudo, adquirido precisamente na campanha 1 . Por isso, nas campanhas 2, 3 e 4 manteve-se o posicionamento de dois dos instrumentos: P11 e P16.

\subsection{Análise dos dados}

Neste trabalho, a análise dos dados recolhidos referese unicamente às campanhas 2,3 e 4, as quais contêm os pontos comuns P11 e P16 necessários para proceder às comparações propostas (ver Figura 2). Assim, os valores 
Tabela 2. Descrição das campanhas de aquisição de dados já realizadas pelo LNEC.

Table 2. Campaigns of data acquisition made by LNEC.

\begin{tabular}{|c|c|c|c|c|}
\hline $\begin{array}{l}\text { Designaçáo da } \\
\text { campanha }\end{array}$ & Início & Fim & No e tipo de sensores usados & $\begin{array}{l}\text { Localizaçáo dos sensores (lat., long., } \\
\text { WGS84) }\end{array}$ \\
\hline Campanha $1^{(*)}$ & $2008 / 05 / 05$ & $2008 / 05 / 07$ & $\begin{array}{l}3 \text { sensores de pressão cablados } \\
\text { Honeywell (P11_0, P14 e P16_0) }\end{array}$ & $\begin{array}{l}\text { Ver pontos a rosa, na Figura } 2 \\
\text { P11_0: } 37^{\circ} 20^{\prime} 55.90^{\prime \prime} \mathrm{N}, 8^{\circ} 50^{\prime} 46.72^{\prime \prime} \mathrm{W} \\
\text { P14: } 37^{\circ} 21^{\prime} 1.19^{\prime} \mathrm{N}, 8^{\circ} 50^{\prime} 50.42^{\prime} \mathrm{W} \\
\text { P16_0: } 37^{\circ} 21^{\prime} 8.10^{\prime \prime N}, 8^{\circ} 50^{\prime} 43.19^{\prime \prime} \mathrm{W}\end{array}$ \\
\hline Campanha 2 & $2009 / 05 / 11$ & $2009 / 05 / 13$ & $\begin{array}{l}1 \text { sensor de pressão autónomo } \\
\text { Infinity_WH (P11) } \\
2 \text { sensores de pressão cablados } \\
\text { Honeywell (P15 e P16) }\end{array}$ & $\begin{array}{l}\text { Ver pontos a vermelho (P15) e a branco } \\
\text { (P11 e P16), na Figura } 2 \\
\text { P11: } 37^{\circ} 20^{\prime} 56.72^{\prime \prime N}, 8^{\circ} 50^{\prime} 47.80^{\prime \prime} \mathrm{W} \\
\text { P15: } 37^{\circ} 21^{\prime} 6.30^{\prime} \mathrm{N}, 8^{\circ} 50^{\prime} 41.78^{\prime \prime} \mathrm{W} \\
\text { P16: } 37^{\circ} 21^{\prime} 6.98^{\prime \prime N}, 8^{\circ} 50^{\prime} 41.39^{\prime \prime W}\end{array}$ \\
\hline Campanha 3 & $2009 / 09 / 07$ & $2009 / 09 / 09$ & $\begin{array}{l}1 \text { sensor de pressão autónomo } \\
\text { Infinity_WH (P11/P11_3) } \\
1 \text { sensor de pressão cablado Honeywell } \\
\text { (P16) }\end{array}$ & 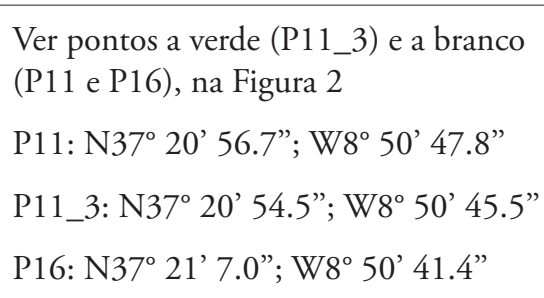 \\
\hline Campanha 4 & $2010 / 09 / 08$ & $2010 / 09 / 10$ & $\begin{array}{l}1 \text { sensor de pressão autónomo } \\
\text { Infinity_WH (P11) } \\
1 \text { sensor de pressão cablado Honeywell } \\
\text { (P16) }\end{array}$ & 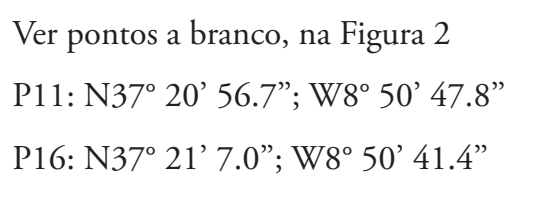 \\
\hline
\end{tabular}

(*) Os valores medidos nesta campanha näo foram considerados neste trabalho.

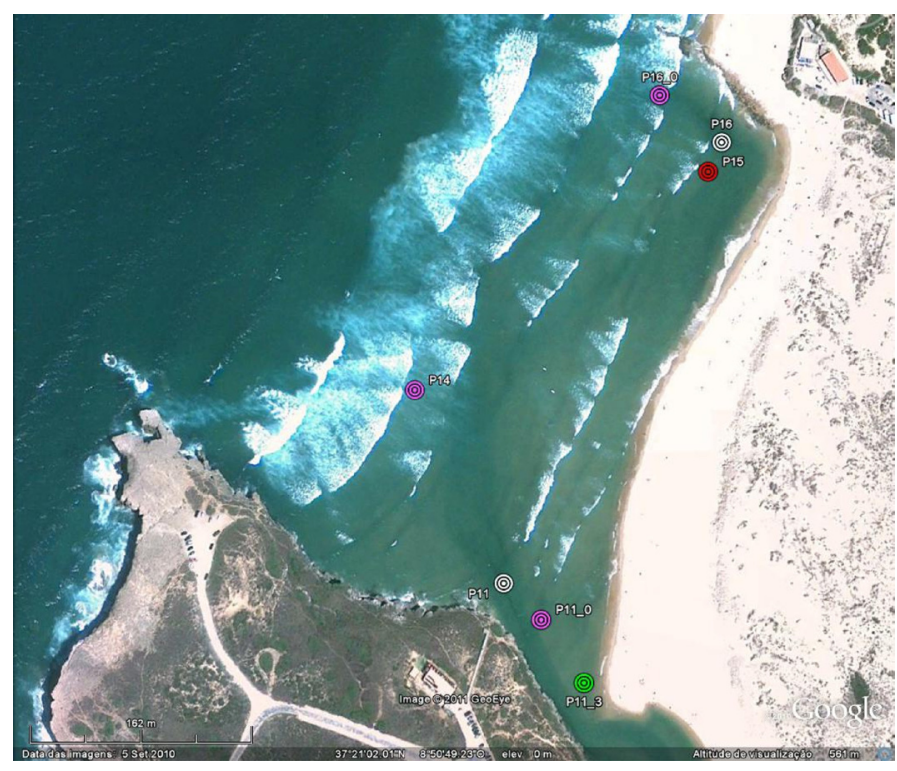

Figura 2. Locais de medição das campanhas \#1, \#2, \#3 e \#4. (Google Earth@).

Figure 2. Location of measuring gages for campaign \#1, \#2, \#3 and \#4. (Google Earth@). medidos na campanha 1 não foram considerados. Para o tratamento dos registos obtidos, foram empregues a análise temporal e a análise espectral dos registos, Bendat e Piersol (1986) e Carvalho (1989).

$\mathrm{Na}$ análise temporal, adoptou-se como critério de definição de ondas o critério de zero ascendente, Goda (1985). Foram, entre outros, calculados os parâmetros altura significativa, HS, i.e., a média do terço de ondas de altura mais elevada do registo, e o período médio, Tmed, i.e., a média dos períodos de onda do registo. $\mathrm{Na}$ análise espectral foram calculados os chamados parâmetros espectrais, com base nos momentos de ordem 0 e 2 do espectro, $m_{0}$ e $m_{2}$, em especial a altura significativa, HM0, e o período médio, TZ. A direcção média das ondas não foi considerada, por limitação dos equipamentos usados. $\mathrm{O}$ tratamento dos registos envolveu o seguinte conjunto de procedimentos:

- Remoção da componente de maré, através do cálculo (aproximado) de uma média móvel, com 2400 pontos, das elevaçôes da superfície livre registadas pelo sensor.

- Obtençáo de registos, $\eta(t)$, maioritariamente de 30 minutos de duração, determinados a partir dos sinais originais, depois de removida a maré; 
- Análise temporal dos registos $\eta(t)$ e obtenção, para cada registo, dos valores de HS (altura significativa) e Tmed (período médio), entre outros;

- Análise espectral dos registos $\eta(t)$, mediante cálculo prévio de estimativas dos seus espectros, usando FFT, e obtenção dos valores da altura significativa, HM0 (equivalente espectral de HS) e do período médio, TZ (equivalente espectral de Tmed), entre outros. Para cada cálculo espectral, de 2048 pontos, foram usados 18000 pontos da série temporal das elevaçóes com frequência de aquisição de $10 \mathrm{~Hz}$. A frequência de corte do espectro considerada foi de $5 \mathrm{~Hz}$.

$\mathrm{Na}$ secção seguinte, discutem-se os resultados, em termos destes parâmetros temporais e espectrais, realizados com base nos registos válidos obtidos nas três campanhas mencionadas $(2,3$ e 4$)$.

\subsection{Resultados das análises aos registos válidos}

Dada a grande quantidade de resultados obtidos, para todos os pontos, optou-se por mostrar nesta secção, como exemplo, apenas a parte de resultados referente ao sensor "Infinity_WH" na posição P11, para as campanhas 2, 3 e 4 (ver Figura 2). Assim, para este local, mostra-se da Figura 3 à Figura 5 os valores de HS (obtidos da análise temporal) e HM0 (obtidos da análise espectral) referentes às três referidas campanhas. Da Figura 6 à Figura 8, mostram-se, para o mesmo ponto e sensor, os correspondentes valores de período médio, Tmed e TZ, para as mesmas campanhas.

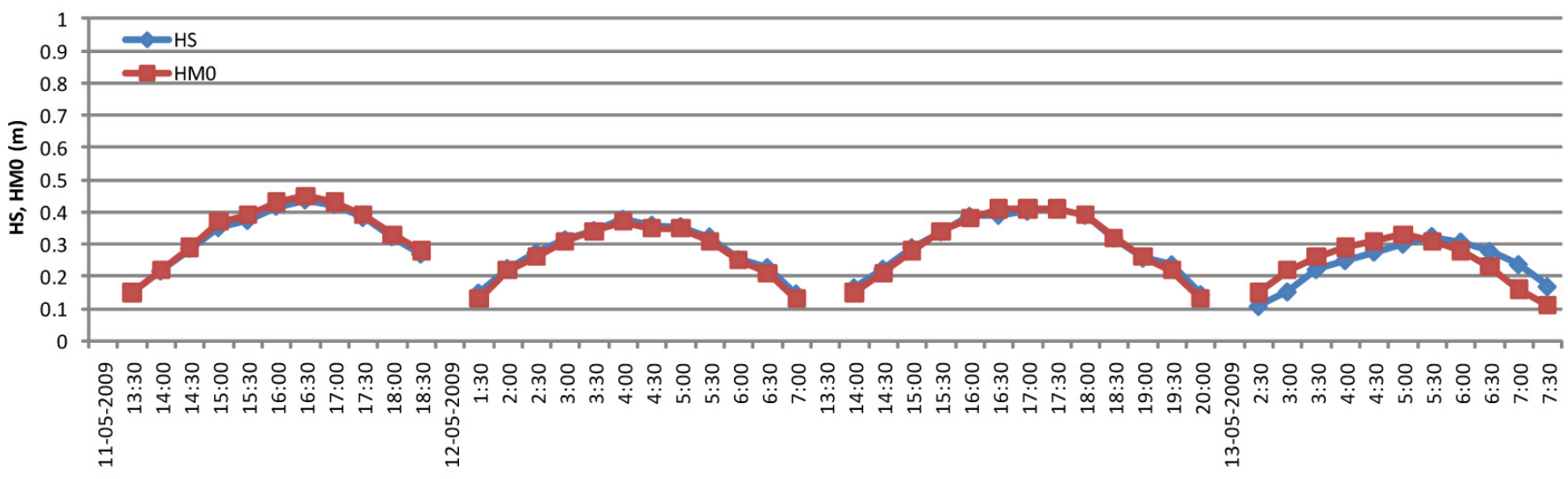

Figura 3. Sensor de pressão "Infinity_WH”. Campanha 2. Alturas significativas, HS e HM0, na posição P11.

Figure 3. "Infinity_WH" pressure transducer. Campaign \#2. Significant wave heights, HS and HMO, at position P11.

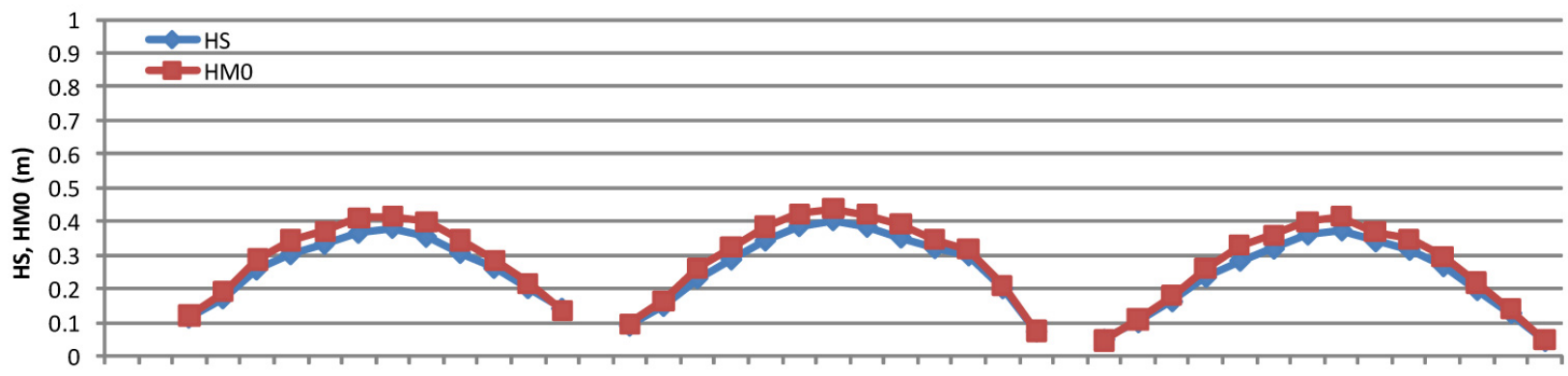

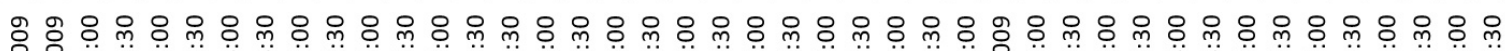

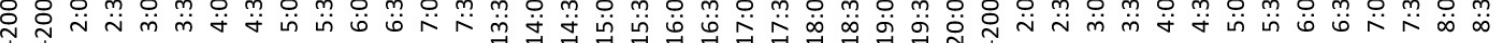
ㅇํㅇ 웅 î.

Figura 4. Sensor de pressão "Infinity_WH”. Campanha 3. Alturas significativas, HS e HM0, na posição P11.

Figure 4. "Infinity_WH" pressure transducer. Campaign \#3. Significant wave heights, HS and HMO, at position P11. 


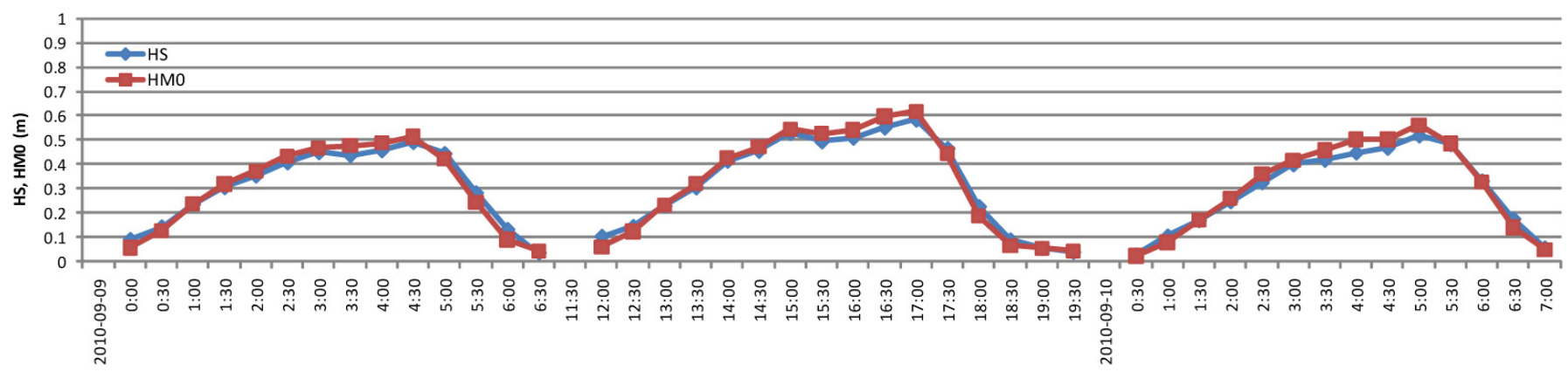

Figura 5. Sensor de pressão "Infinity_WH”. Campanha 4. Alturas significativas, HS e HM0, na posição P11.

Figure 5. "Infinity_WH" pressure transducer. Campaign \#4. Significant wave heights, HS and HMO, at position P11.

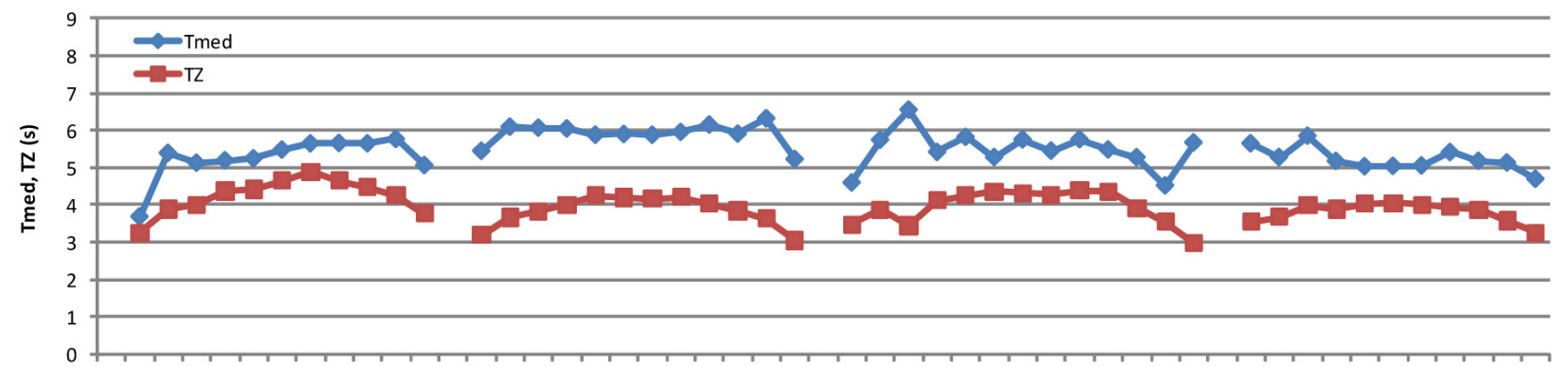

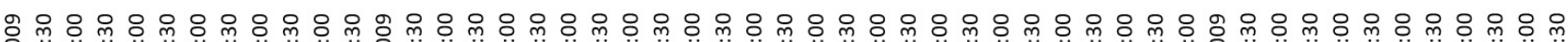

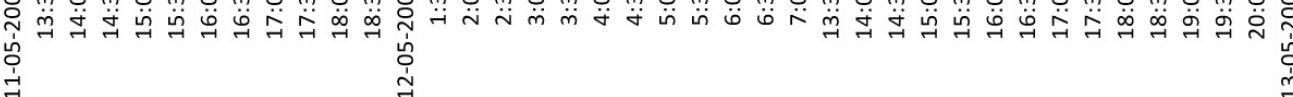

Figura 6. Sensor de pressão “Infinity_WH”. Campanha 2. Períodos médios, Tmed e TZ, na posição P11.

Figure 6. "Infinity_WH" pressure transducer. Campaign \#2. Mean wave periods, Tmed and TZ, at position P11.

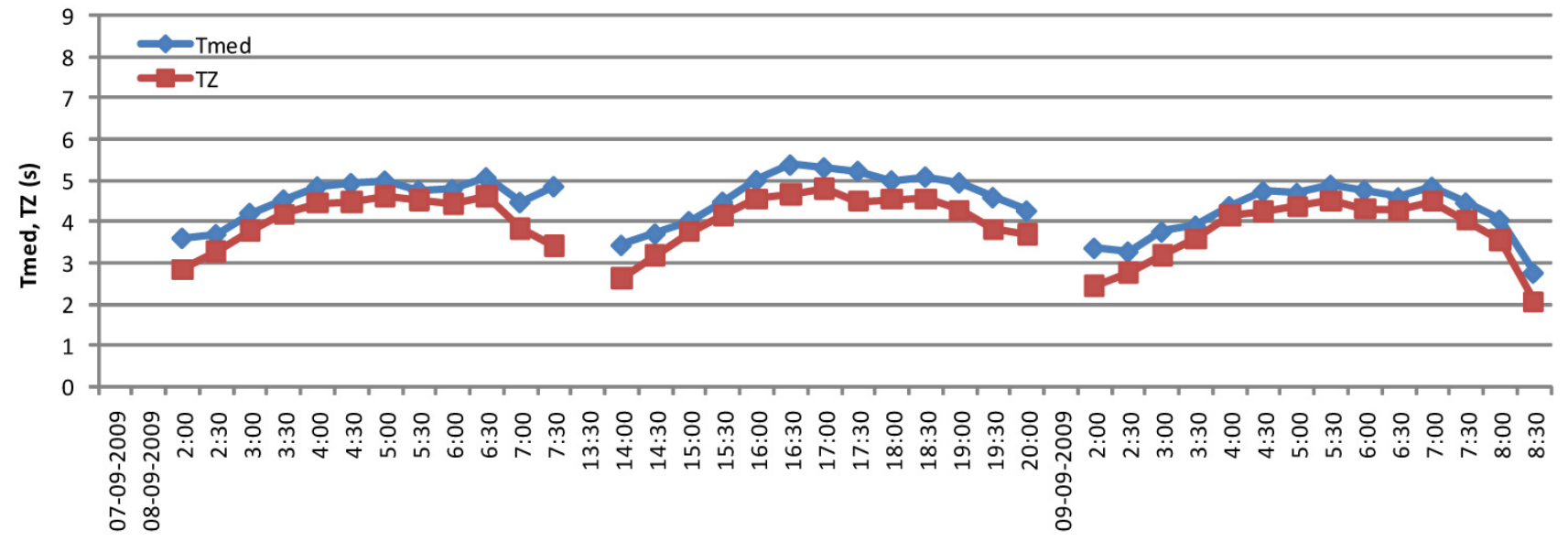

Figura 7. Sensor de pressão "Infinity_WH”. Campanha 3. Períodos médios, Tmed e TZ, na posição P11.

Figure 7. "Infinity_WH" pressure transducer. Campaign \#3. Mean wave periods, Tmed and TZ, at position P11. 


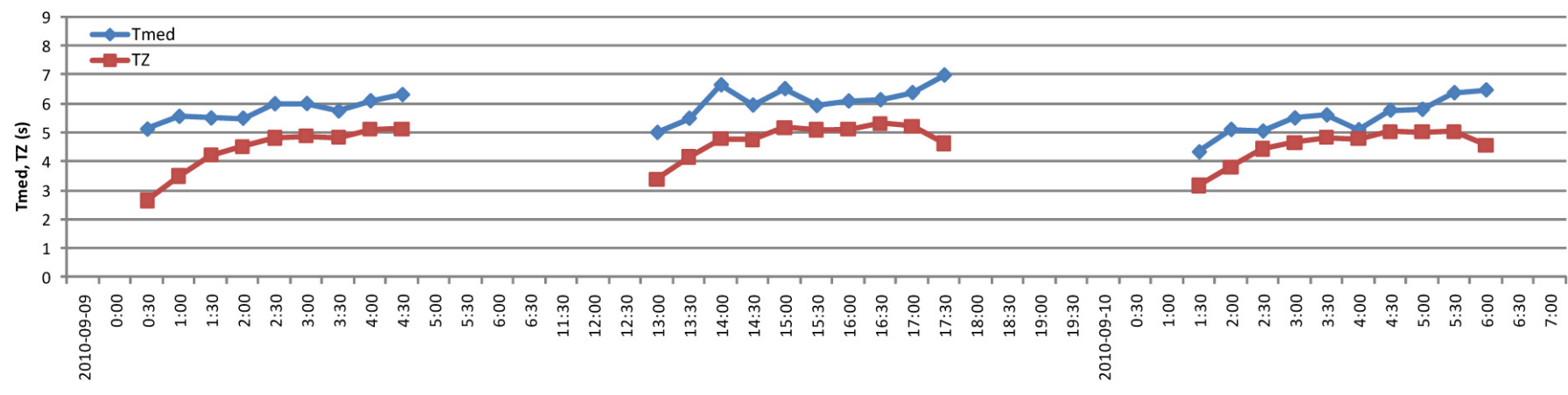

Figura 8. Sensor de pressão "Infinity_WH”. Campanha 4. Períodos médios, Tmed e TZ, na posição P11.

Figure 8. "Infinity_WH" pressure transducer. Campaign \#4. Mean wave periods, Tmed and TZ, at position P11.

Dos resultados obtidos nas análises temporal e espectral, de todos os registos válidos das campanhas 2,3 e 4, para todos os pontos de medição (P11, P15 e P16), retiram-se as seguintes conclusóes:

- Em qualquer das campanhas e para qualquer dos instrumentos de medição, os valores de HS e HM0 são muito idênticos, isto é, as análises temporal e espectral produzem valores muito próximos, o que era esperado;

- Em todas as campanhas, verifica-se, porém, que os valores do período médio dos registos obtidos com a análise espectral (TZ) são sempre inferiores aos obtidos com a análise temporal (Tmed). A razão para esta diferença reside na fórmula de cálculo usada no método espectral, que, implicitamente (através dos momentos de ordem zero e dois do espectro), considera todas as ondas existentes no registo, por mais pequenas que sejam, ao passo que o método directo, empregue na análise temporal, considera apenas ondas que cruzam o nível médio no sentido ascendente, não considerando portanto as pequenas oscilaçóes, que ocorrendo acima ou abaixo desse nível, não o cruzam. Apesar disso, para qualquer dos instrumentos de medição, o andamento dos valores de Tmed e TZ é semelhante, principalmente para as campanhas 2 e 3 ;

- A limitação da altura da onda devida à profundidade é nítida ao observarem-se os grupos de mediçóes das figuras anteriores,

- Em todas as campanhas, verificaram-se estados de agitação pouco energéticos associados a períodos de onda baixos: os valores de HS (HM0) não excedem $0.5 \mathrm{~m}$ nas campanhas 2 e 3 e $0.7 \mathrm{~m}$ na campanha 4 . Os períodos de onda médios são também bastante baixos, não excedendo $5.5 \mathrm{~s}$ (em TZ) e $7 \mathrm{~s}$ (em Tmed).

Como é referido atrás, este conjunto de dados é demasiado escasso para definir adequadamente um regime de agitação marítima ou para avaliar os efeitos da agitação na morfologia do sistema a longo-prazo. Apesar disso, estes resultados podem ser de grande interesse para a calibração de modelos numéricos de propagação de ondas, como é o caso do modelo SWAN.

\section{SIMULAÇÓES NUMÉRICAS}

Nesta secção, descreve-se a aplicação efectuada com o modelo SWAN para a zona em estudo com vista à simulação das condições de agitação marítima que se verificaram nos dias das campanhas 2 , 3 e 4 . Para cada período de campanha, seguiu-se o seguinte procedimento:

- Utilização como condiçóes ao largo do SWAN as fornecidas pelo modelo de geração WAVEWATCH III, WWIII (Tolman, 1999, 2002);

- Cálculo com o modelo SWAN das características das ondas (HS, TZ, DIR) em pontos tâo perto quanto possível dos locais de medição P11 e P16;

- Comparaçáo das estimativas numéricas dos valores de HS e TZ, produzidas com o SWAN, com os valores medidos in situ, nas posiçóes P11 e P16.

\subsection{O modelo SWAN}

O modelo SWAN, Booij et al. (1999), acrónimo de Simulating WAves Nearshore é um modelo numérico para geração, propagação e dissipação da agitação marítima, baseado na equação para a conservação da acção de onda. Trata-se de um modelo de domínio público (freeware), em constante desenvolvimento pela Delft University of Technology da Holanda, que possui como uma das maiores vantagens a manutenção da estrutura dos ficheiros de dados e de resultados o que permite a fácil actualização de versóes mais robustas e completas do modelo sempre que necessário.

Este modelo propaga a agitação marítima desde o largo até próximo da costa considerando os processos físicos de refracção, difracção e empolamento devido a variaçóes do fundo e presença de correntes, crescimento de onda por acção dos ventos, rebentação por influência do fundo e por excesso de declividade (whitecapping), dissipação de energia devido à fricção do fundo, bloqueio e reflexão por correntes opostas e transmissão através de obstáculos.

O campo de ondas na zona é caracterizado pelo espectro bidimensional de densidade da acção da agitação marítima. Com esta representação, é possível a aplicação do modelo em áreas onde o crescimento da agitação marítima pela acção do vento seja notável ou onde estados de mar, ou mesmo ondulação, estejam presentes. A propagação da agitação, 
nos modos estacionário ou não estacionário, nos espaços geográfico e espectral, é realizada utilizando esquemas numéricos implícitos. A zona em estudo pode ser descrita com coordenadas cartesianas ou esféricas, utilizando uma malha "rectangular".

Os dados necessários para a execução do SWAN são a malha batimétrica da zona a modelar e as condiçóes de agitação na fronteira de entrada do domínio, para além de um conjunto de outros parâmetros de cálculo. De entre os vários resultados obtidos pelo SWAN destacam-se a altura significativa, os períodos de pico e médio, as direcçóes de pico e média, a dispersão direccional, o parâmetro de largura de banda e nível de água em qualquer parte do domínio computacional.

De entre as limitaçóes do modelo referem-se as seguintes:

- A calibração de muitos dos parâmetros envolvidos na descrição dos diferentes fenómenos físicos no SWAN baseou-se em dados da campanha JONSWAP, realizada no Mar do Norte (Hasselmann et al., 1973). Tais parâmetros poderão não estar correctos para áreas com diferentes características de clima de ondas (ondas associadas ao estado de mar vaga e ondulação) ou para diferentes características do fundo marinho;

- A difracção no SWAN é modelada de modo simples como uma dispersão direccional, o que pode constituir a sua principal limitação, além de não considerar os efeitos de espalhamento de Bragg;

- A inclusão da difracção nos cálculos numéricos implica que o espaçamento da malha computacional, relativamente ao comprimento de onda, seja tal que garanta a convergência dos cálculos computacionais. Isso implica, por vezes, que as malhas sejam de tal dimensão que pode inviabilizar a execução dos cálculos.

\subsection{Condiçóes ao largo}

Consideraram-se como condiçóes ao largo os estados de mar ao largo estimados pelo modelo de $3^{a}$ geraçáo
WAVEWATCH III (GODAE, 2010). Estas estimativas contêm os parâmetros de onda seguintes: HS (altura de onda significativa), TZ (período médio de onda) e DIR (direcção de onda), obtidas a cada três horas, referentes ao período de cada campanha (Campanha 2- de 11 a 13 de Maio de 2009; Campanha 3 - de 7 a 9 de Setembro de 2009; Campanha 4 - de 7 a 10 de Setembro de 2010), para os pontos da malha $38 \mathrm{~N} 10 \mathrm{~W}$ e $37 \mathrm{~N} 10 \mathrm{~W}$, seleccionados por serem os pontos disponíveis mais próximos da Praia da Amoreira, ver Figura 9. Uma vez conhecidas as estimativas do estado do mar ao largo, caracterizadas pela sua altura de onda significativa, HS, período de pico, TP, e direcção de pico, DIR, o modelo SWAN é capaz de transferir esses valores para a praia da Amoreira.

\subsection{Domínio computacional e condiçóes do modelo SWAN}

O domínio computacional do modelo SWAN foi discretizado mediante a utilização de três malhas rectangulares, Figura 9. A malha mais larga ( $1^{\text {a }}$ malha $-1^{\text {st }}$ Mesh) tem dimensōes $60 \mathrm{~km}$ x $20 \mathrm{~km}$ e resolução $500 \mathrm{~m}$. A malha média ( $2^{\text {a }}$ malha $-2^{\text {nd }}$ Mesh) tem dimensões $8 \mathrm{~km} \times 3 \mathrm{~km}$ e resolução $50 \mathrm{~m}$. A malha mais fina ( $3^{\mathrm{a}}$ malha $-3^{\text {rd }}$ Mesh) tem dimensôes $1.2 \mathrm{~km} \times 1 \mathrm{~km}$ e resolução $5 \mathrm{~m}$.

Para este estudo, foram usadas as seguintes batimetrias:

- Carta hidrográfica no 6, escala 1/150 000, Costa Portuguesa, do Cabo de Sines ao Cabo de S. Vicente, do Instituto Hidrográfico;

- Cartas batimétricas referentes às campanhas de Maio de 2009 (Campanha 2) e de Setembro de 2009 (Campanhas 3 e 4$)$;

De acordo com alguns testes de sensibilidade efectuados com o modelo SWAN, destinados a analisar os parâmetros óptimos de rebentação (variando o coeficiente de 0.5 a 0.8 ) e de atrito de fundo (variando o coeficiente de 0.2 a 0.5 ), e o tipo de espectro em frequência (JONSWAP ou GAUSS), chegou-se à seguinte configuração para execuçáo do modelo:
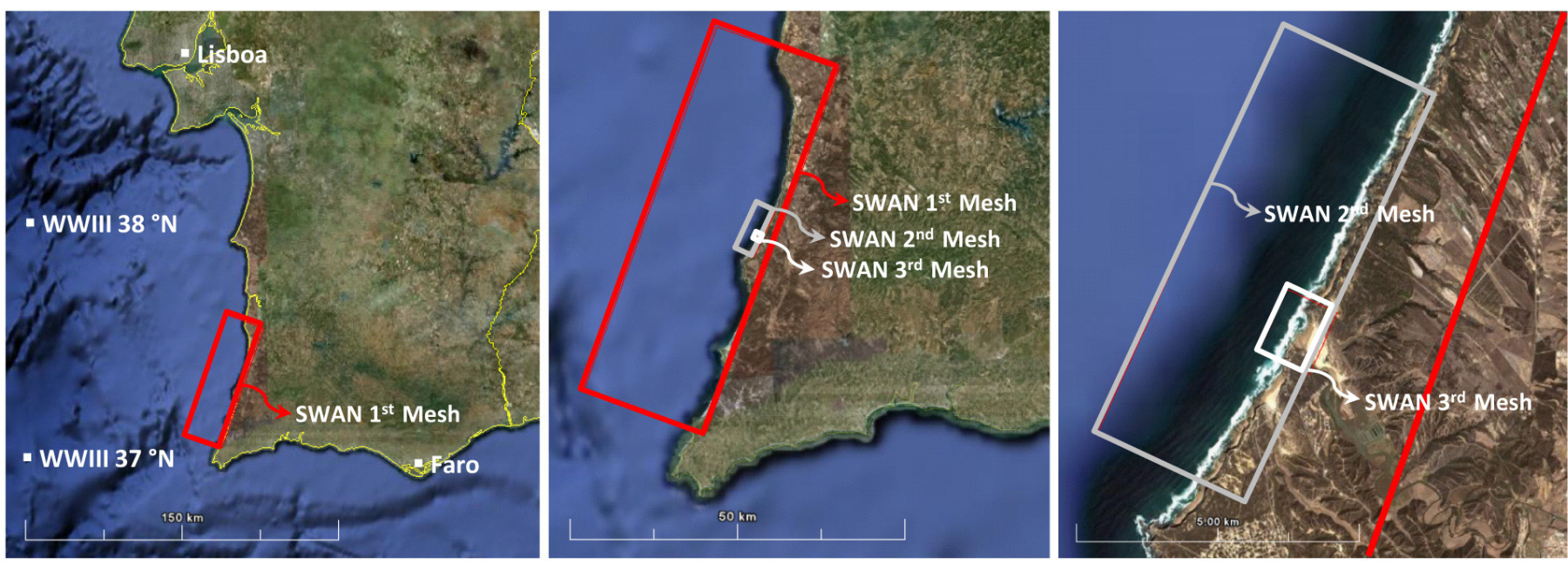

Figura 9. Pontos WWIII (esquerda) e domínios computacionais SWAN (centro e direita). Figure 9. WWIII points (left) and SWAN computational domains (center and right). 
- Foi utilizada a versão SWAN 40.72, em modo estacionário, sem correntes e sem ventos;

- Foi definido um espectro direccional a partir da discretização de um espectro em frequência contendo 21 intervalos, de 0.07 a $2.0 \mathrm{~Hz}$, com distribuição logarítmica, e uma discretização em direcção cobrindo $270^{\circ}$, dividida em 21 intervalos $\left(\Delta=10^{\circ}\right)$;

- Foi considerado um nível de maré variando de hora-a-hora ao longo da praia de acordo com os valores observados em Sines (cerca de $65 \mathrm{~km}$ a Norte do local de estudo) para os períodos em estudo;

- Foi adoptado um coeficiente de rebentaçáo constante: 0.65 para Maio de 2009, 0.8 para Setembro de 2009 e 0.65 para Setembro de 2010;

- Foi usada a lei de Madsen, adoptando como parâmetro de atrito de fundo o valor de 0.05 .

É de notar que as condições de ondas impostas na fronteira da $1^{\text {a }}$ malha do modelo SWAN foram as correspondentes aos resultados do modelo WWIII no ponto $38 \mathrm{~N} 10 \mathrm{~W}$, uma vez que não se verificaram diferenças substanciais nos valores obtidos pelo SWAN entre os resultados para aquele ponto e os resultados para o ponto $37 \mathrm{~N} 10 \mathrm{~W}$.

\subsection{Estimativas do modelo WWIII para o ponto ao largo $38 \mathrm{~N} 10 \mathrm{~W}$}

Com base nas condiçóes acima enunciadas, o conjunto de simulaçóes efectuadas com o modelo SWAN incluiu os três dias de medição de Maio de 2009 (11-13), os três dias de Setembro de 2009 (7-9), e os quatro dias de Setembro de 2010 (7-10), considerando as condiçóes das ondas ao largo definidas pelo modelo WWIII nos limites W, N e $S$ da primeira malha, para o ponto $38 \mathrm{~N} 10 \mathrm{~W}$ - Figura 9.

Apesar de este ponto se encontrar relativamente longe da $1^{\text {a }}$ malha, devido às características das ondas, especialmente devido aos seus curtos períodos, não é expectável que ocorram diferenças significativas entre o largo e a fronteira da $1^{\text {a }}$ malha. Os valores de HS, TP e DIR para esses dias encontram-se representados na Figura 10.

Embora os períodos das ondas sejam semelhantes (na gama entre 6 s e 9 s), as alturas de onda foram mais elevadas nos períodos 11 a 13 de Maio de 2009 e de 8 a 10 de Setembro de 2010 , com valores de cerca de $2.0 \mathrm{~m}$, do que no período de 7 a 9 de Setembro de 2009 (valores aproximadamente de $1.5 \mathrm{~m}$ ). Os períodos de pico variaram nos três períodos analisados entre $6 \mathrm{~s}$ e $9 \mathrm{~s}$. Quanto às direcçóes de onda, enquanto no período de 11 a 13 de Maio de 2009 os valores variaram entre $245^{\circ}$ e $310^{\circ}$, nos restantes períodos os valores da direcção apresentam valores acima de $300^{\circ}$ e, portanto, dispóem-se de forma mais perpendicular à batimetria da zona em estudo, atingindo-a mais frontalmente.

As simulaçōes foram executadas hora-a-hora, pelo que foram efectuados $3 \times 24=72$ cálculos para os períodos considerados. Como os valores do modelo WWIII são disponibilizados a cada 3 horas, assumiu-se um estado de mar estacionário nesse período.

\subsection{Resultados do modelo SWAN}

Os resultados do modelo SWAN em qualquer ponto do domínio computacional são a altura significativa (HS), o período médio (TZ), o período de pico (TP), a direcção de pico (DIR), o comprimento de onda (L) e a dispersão direccional. Para este trabalho, foram obtidos valores de HS para as posiçóes P11, P15 e P16 (ver Figura 2).

Para ilustrar este procedimento, a Figura 11 mostra os resultados do modelo nas seguintes situaçôes: a) 12 de Maio 2009 - 16:00 $\left(\mathrm{HS}=1.78 \mathrm{~m}, \mathrm{TP}=7.53, \mathrm{DIR}=310^{\circ}\right)$; b) 9 de Setembro $2009-21: 00(\mathrm{HS}=1.35 \mathrm{~m}, \mathrm{TP}=6.82 \mathrm{~s}$, DIR $=327^{\circ}$ ); c) 9 de Setembro $2010-16: 00$ (HS=1.72 m, $\mathrm{TP}=8.5 \mathrm{~s}, \mathrm{DIR}=313^{\circ}$ ).

Como pode ser observado nessa figura, há uma diminuição da altura de onda à medida que a onda se propaga em direcção à praia. Essa variação é mais significativa nas campanhas 2 e 4, que apresentam uma maior altura de onda ao largo e direcções de onda ao largo idênticas. Com efeito, para essas duas campanhas, o padrão das isolinhas de altura de onda é bastante semelhante, ao contrário do que se verifica para a campanha 3, dados o menor valor de altura ao largo e a direcção de onda de $327^{\circ}$.

\section{COMPARAÇÃO DE RESULTADOS}

A comparação entre os resultados numéricos e os valores medidos, efectuada para as três campanhas de campo, é apresentada e discutida nesta secção.

Assim, da Figura 12 à Figura 14 comparam-se os valores dos parâmetros de altura de onda significativa medidos (HS_meas) nas três campanhas com os respectivos valores dos parâmetros estimados numericamente com o modelo SWAN (HS_num_SWAN), para as condiçóes referidas, nos pontos P11 e P16.

$\mathrm{Na}$ Tabela 3 apresentam-se parâmetros estatísticos, tais como o erro médio quadrático, RMSE (m), e o erro médio absoluto, Viés (m), que permitem, de forma quantitativa, avaliar a concordância (ou não) entre os valores medidos e estimados numericamente. Um outro parâmetro deste género presente nesse quadro é o chamado índice de concordância, ic, ou índice de Willmott, dado por:

$$
i c=1-\frac{\sum_{i=1}^{n}\left|y_{i}-x_{i}\right|^{2}}{\sum_{i=1}^{n}\left(y_{i}-\bar{x}|+| x_{i}-\bar{x} \mid\right)^{2}}
$$

onde $x_{i}$ são os valores medidos (referência), $y_{i}$ são os valores dos modelos numéricos, $\bar{x}$ é a média do valor experimental e $n$ é o número de pontos. 

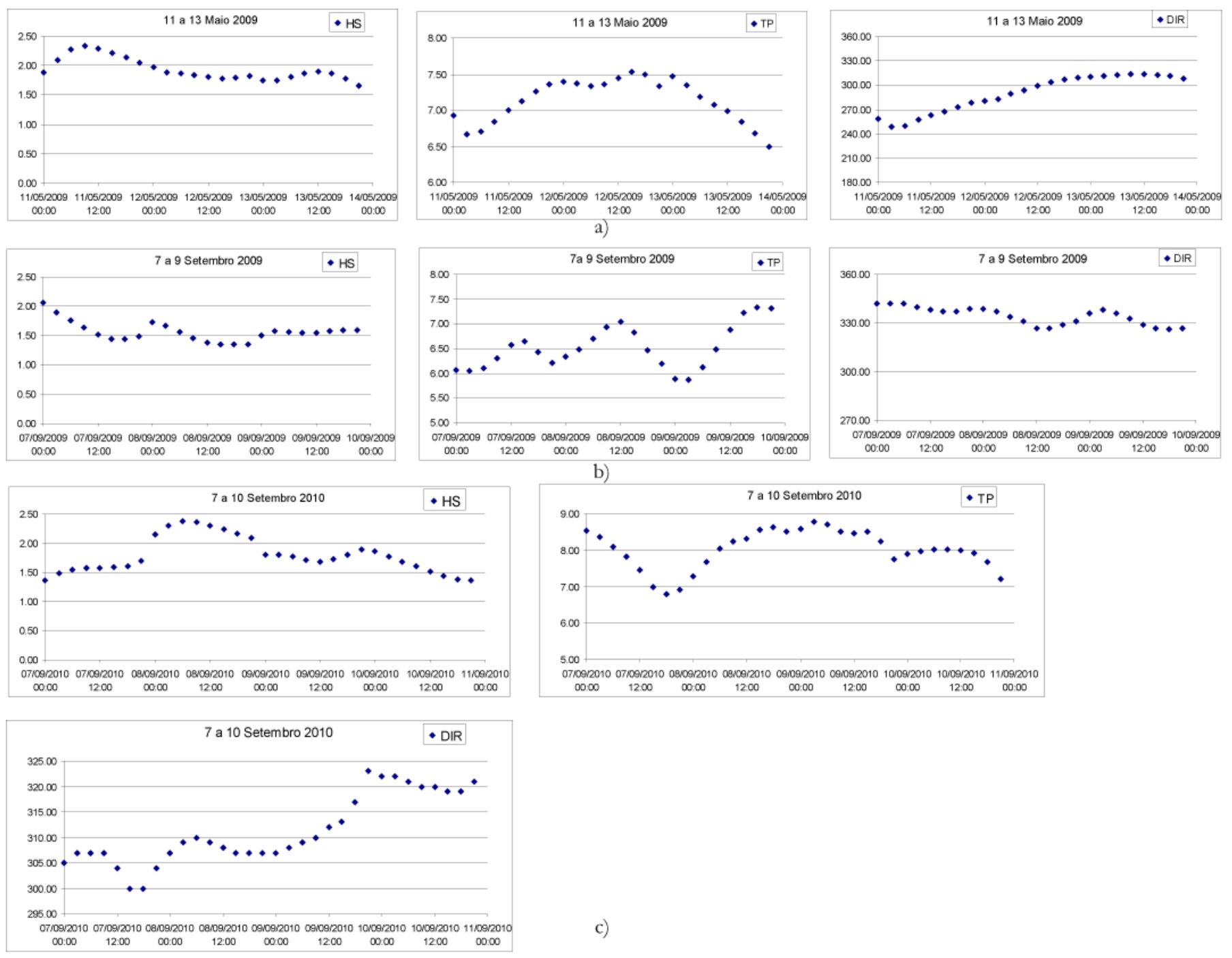

c)

Figura 10. Resultados (HS, TP e DIR) do modelo WWIII no ponto 38N10W, a) de 11 a 13 de Maio de 2009; b) de 7 a 9 de Setembro de 2009 e c) de 7 a 10 de Setembro de 2010.

Figure 10. WWIII results (HS, TP and DIR) at point 38N10W on a) May (11 to 13); b) September (7 to 9) 2009 and c) September (7 to 10) 2010.
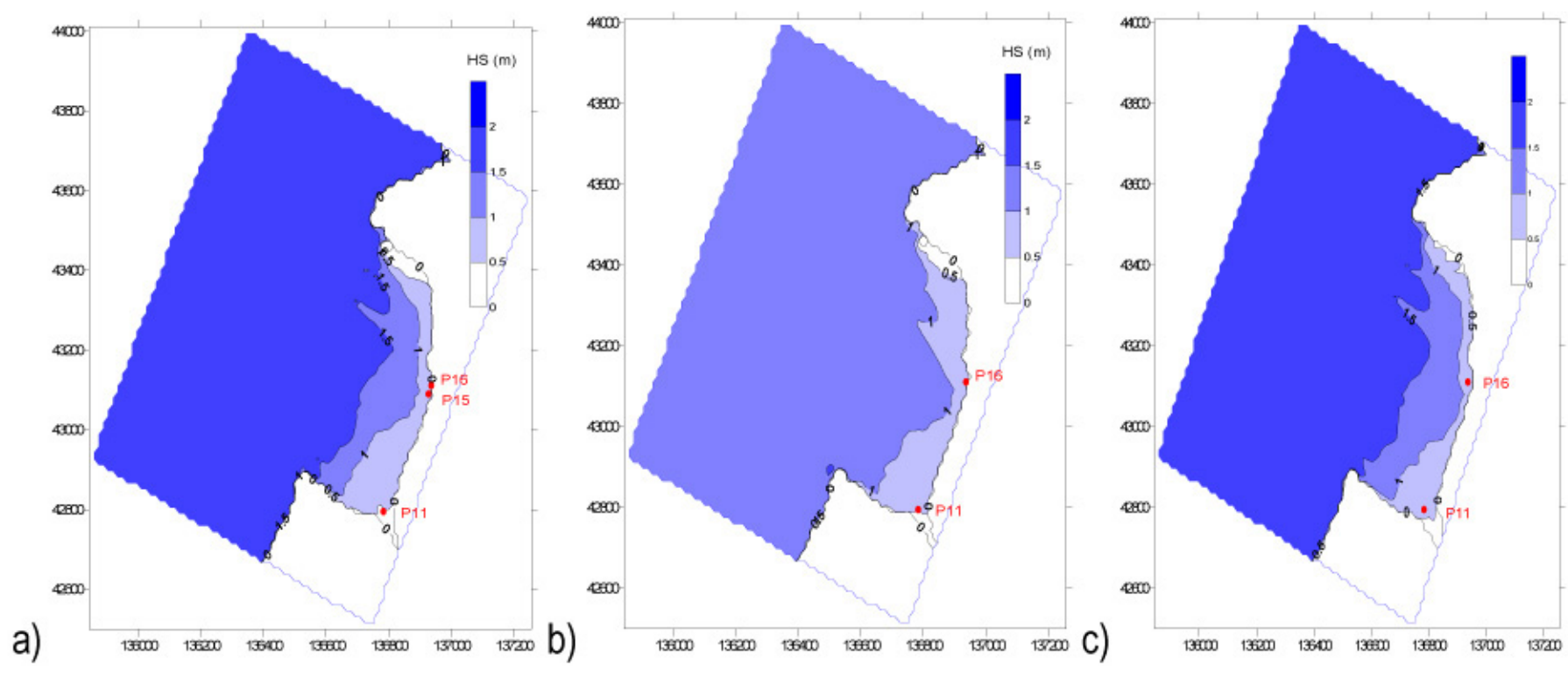

Figura 11. Resultados SWAN para HS. a) 12 Maio 2009, 16:00, b) 9 Setembro 2009, 21:00 e c) 9 de Setembro 2010, 16:00.

Figure 11. SWAN results. HS as of a) May 12 2009, 16:00; b) September 9 2009, 21:00; c) September 9 2010, 16:00. 

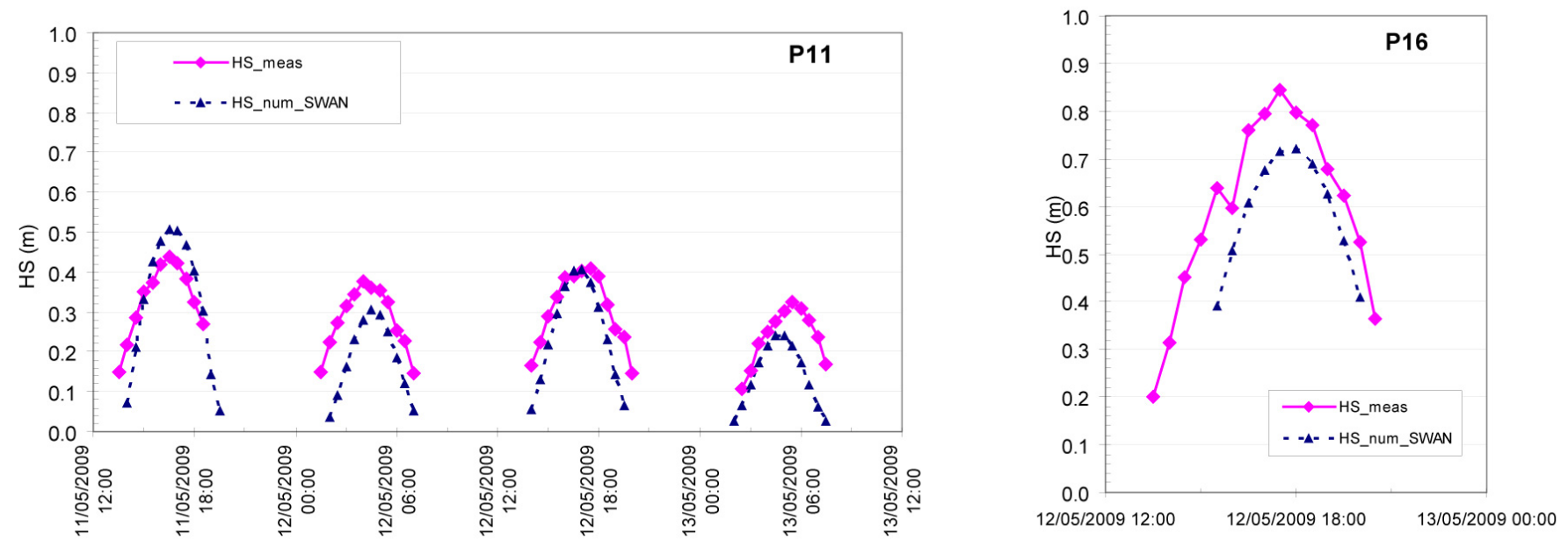

Figura 12. Campanha 2. Comparação entre HS_meas e HS_num_SWAN em P11 e P16.

Figure 12. Campaign \#2. Comparison between HS_meas and HS_num_SWAN at P11 and P16.
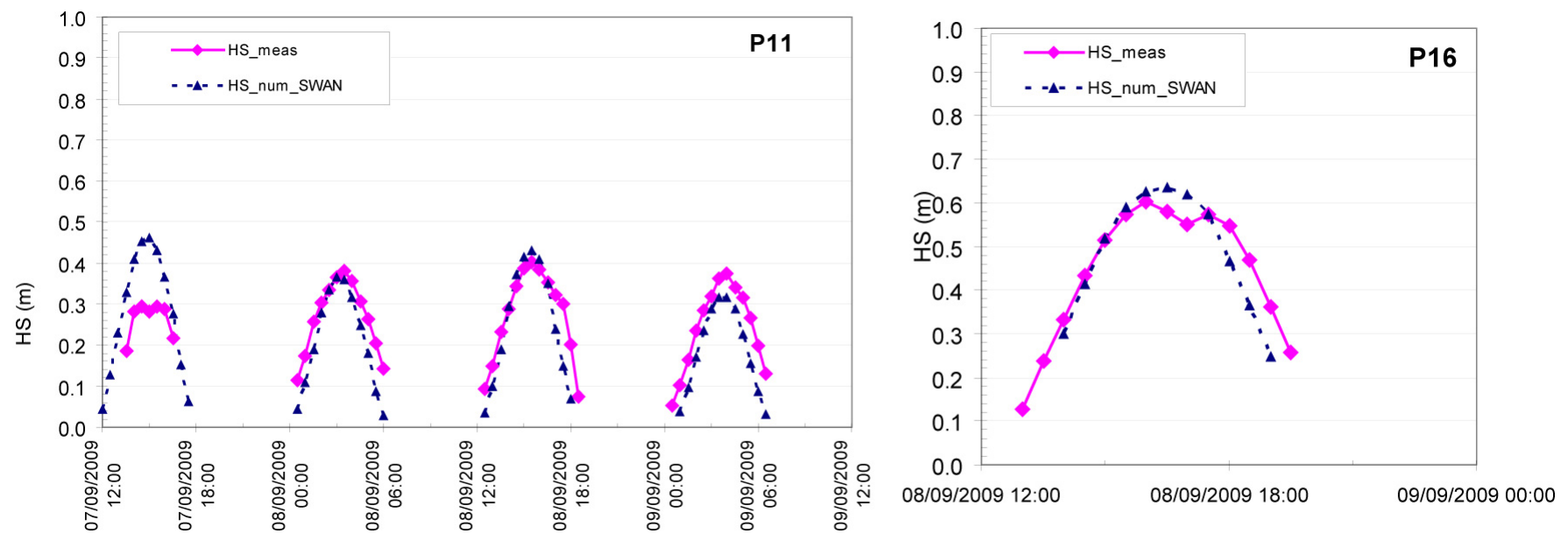

Figura 13. Campanha 3. Comparação entre HS_meas e HS_num_SWAN em P11 e P16.

Figure 13. Campaign \#3. Comparison between HS_meas and HS_num_SWAN at P11 and P16.
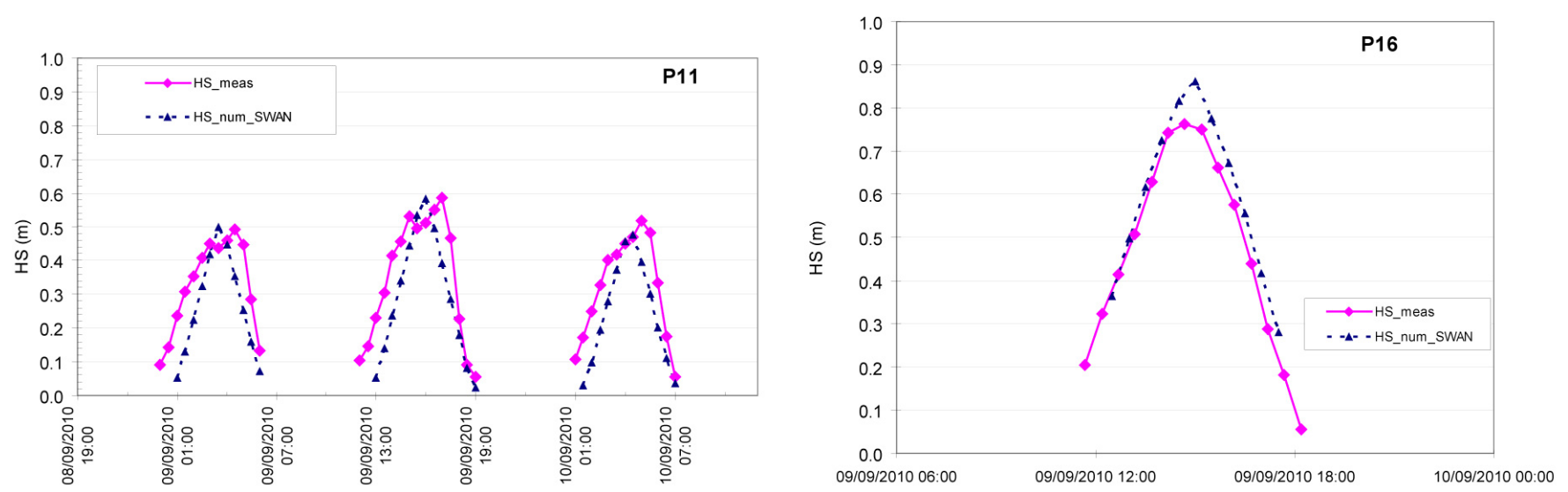

Figura 14. Campanha 4. Comparação entre HS_meas e HS_num_SWAN em P11 e P16.

Figure 14. Campaign \#4. Comparison between HS_meas and HS_num_SWAN at P11 and P16. 
Tabela 3. Parâmetros estatísticos para as alturas de onda.

Table 3. Statistical parameters for wave heights.

\begin{tabular}{c|c|c|c|c|c|c}
\hline \multirow{2}{*}{ Local/Campanha no $^{*}$} & \multicolumn{3}{|c|}{ P11 } & \multicolumn{3}{c}{ P16 } \\
\cline { 2 - 7 } & 2 & 3 & 4 & 2 & 3 & 4 \\
\hline Viés (m) & -0.063 & -0.024 & -0.099 & -0.117 & -0.016 & 0.057 \\
\hline RMSE (m) & 0.097 & 0.084 & 0.125 & 0.129 & 0.060 & 0.085 \\
\hline ic & 0.95 & 0.95 & 0.96 & 0.98 & 0.99 & 0.98 \\
\hline
\end{tabular}
que:

Da análise das figuras e quadro anteriores, verifica-se

- A tendência (andamento) da altura de onda significativa estimada com o modelo numérico SWAN é muito semelhante à tendência dos valores medidos in-situ, para as posiçóes P11 e P16, em qualquer das campanhas. Por outro lado, verifica-se que os valores numéricos estão muito próximos dos valores medidos, sendo o desvio máximo (Viés) de $0.117 \mathrm{~m}$. Os valores de RMSE são inferiores a $0.129 \mathrm{~m}$ e os índices de concordância (ic) superiores a 0.95 , em qualquer das campanhas e posiçōes, o que mostra boa concordância entre as séries de valores em comparação;

- De uma forma geral, o modelo numérico produz valores inferiores aos valores medidos. Esse facto é mais notório para a posição P11, para a qual se verificou sempre uma subestimação dos valores numéricos face aos medidos em todas as campanhas. Para a posição P16, isso acontece apenas para as campanhas 2 e 3 ;

- Para todos os casos, as maiores diferenças ocorrem para a posição P11 (o índice de concordância ic é da ordem de 0.95$)$. É de salientar que a posição P11 se refere a um local, quase no meio da ribeira de Aljezur, onde a profundidade da água é muito pequena. Notar também que os fenómenos de correntes e de fluxo de maré, que ocorrem na natureza, não são aqui simulados com o modelo SWAN, limitaçôes estas que podem explicar algumas das diferenças observadas. Notar que no âmbito das campanhas de campo referidas, para a área em estudo, foram apenas obtidos dados de correntes e de fluxo de maré muito localizados na zona da embocadura da ribeira de Aljezur, claramente insuficientes para as simulaçôes numéricas realizadas neste trabalho. Apenas o recurso a modelos regionais de circulação hidrodinâmica poderão permitir adequada caracterização daqueles fenómenos na zona marítima local.

\section{CONCLUSÓES}

Nesta comunicação, descreveram-se as medições, a análise dos dados medidos e a aplicaçáo efectuada com o modelo SWAN com vista a caracterizar o padráo de ondas geradas pelo vento para a zona da embocadura da Ribeira de Aljezur, na praia da Amoreira, Aljezur.

Assim, foram abordados os procedimentos de preparação e de aquisição de dados adoptados nas quatro campanhas de medições aí efectuadas e descreveram-se os procedimentos de análise efectuados aos registos medidos em três dessas campanhas (2, 3 e 4), assim como os procedimentos de utilização do modelo numérico SWAN usado para estimar o campo de ondas local.

Dos resultados das análises temporal e espectral efectuadas aos registos válidos das mediçóes efectuadas nas campanhas 2, 3 e 4, para todos os pontos de medição (P11, P15 e P16), verificou-se que, para qualquer das campanhas e para qualquer dos instrumentos de medição, o andamento dos valores de HS e HM0 é muito idêntico, isto é, as análises temporal e espectral produzem valores muito próximos, o que era esperado. Porém, verificou-se também que os valores do período médio dos registos obtidos com a análise espectral (TZ) são sempre inferiores aos obtidos com a análise temporal (Tmed). Nas figuras referentes à altura significativa, observam-se periodicidades que assinalam a nítida limitação das alturas da onda devido às baixas profundidades a que se efectuaram as mediçóes. Os dados medidos, apesar de escassos para definir adequadamente um regime de agitação marítima ou para avaliar os efeitos da agitação na morfologia do sistema a longo-prazo, por estarem associados a condiçóes locais (nomeadamente de profundidade) muito peculiares, podem claramente servir para a calibração de modelos numéricos de propagação de ondas, como é o caso do modelo SWAN.

Considerando unicamente os dados produzidos no âmbito dessas três campanhas de recolha de dados, podese concluir que, para o cálculo das alturas de onda, a modelação numérica produz resultados de muito boa qualidade, pelo que se considera que o modelo SWAN é adequado para prever as alturas de onda que se verificam na praia da Amoreira. As diferenças observadas nos resultados apresentados anteriormente devem-se principalmente a algumas simplificaçóes consideradas na aplicação do modelo numérico:

- Devido à falta de informação, as simulações SWAN foram feitas apenas para condiçōes ao largo referentes ao ponto $38 \mathrm{~N} 10 \mathrm{~W}$;

- Os dados de maré não são exactamente locais mas referem-se a local relativamente distante: Porto de Sines, $65 \mathrm{~km}$ a norte do local de estudo;

- Nas simulações, não foram considerados vento e correntes;

- P11 está localizado na entrada da ribeira de Aljezur, 
pelo que é fortemente influenciado pelo fluxo gerado por correntes locais, condiçôes náo consideradas pelo modelo.

A estes erros, essencialmente numéricos, juntam-se os erros inerentes às próprias mediçóes in-situ, como são os que, por exemplo, ocorrem em condiçôes de águas pouco profundas e em casos em que existem correntes (ambos são aplicáveis), ver Lee e Wang (1984) e Bishop e Donelan (1987).

Finalmente, como trabalho futuro, aponta-se a necessidade de incluir ventos e correntes nas simulaçóes a efectuar com o modelo SWAN, o que poderá ser concretizado com o recurso a modelos regionais de circulação hidrodinâmica.

\section{AGRADECIMENTOS}

Os autores agradecem o apoio do projecto MADyCOS (Multidisciplinary integrated Analysis of the sediment Dynamics and fecal contamination in intermittent Coastal Systems), financiado pela Fundaçáo para a Ciência e a Tecnologia (contrato PTDC/ECM/66484/2006), que financiou este trabalho. Agradecem também ao projecto FCT BayBeach (PTDC/CTE-GEX/66893/2006), que facultou os dados batimétricos usados na aplicação do modelo SWAN.

\section{BIBLIOGRAFIA}

Bendat, J.S.; Piersol, A.G. (1986) - Random Data. Analysis and Measurement Procedures. 2nd Edition, 566p., John Wiley \& Sons, New York, U.S.A. ISBN: 0-471-04000-2

Bishop, C.; Donelan, M. (1987) - Measuring waves with pressure transducers. Coastal Engineering, 11(4):309328. DOI:10.1016/0378-3839(87)90031-7.

Booij, N.; Ris, R.C.; Holthuijsen, L.H. (1999) - A third-generation wave model for coastal regions, Part I, Model description and validation. Journal of Geophysical Research, 104(C4):7649-7666. DOI:10.1029/98JC02622.

Capitão, R.; Fortes, C.J.E.M.; Carvalho, F.; Coli, A.B.; Pinheiro, L.V. (2006) - Wave Regime Characterization on thePortugueseCoastusing Hindcastand WavePropagation Models. Coastal Engineering 2006: Proceedings of the 30th International Conference on Coastal Engineering, 1:603-615. DOI:10.1142/9789812709554_0052.

Capitấ, R.; Fortes, C.J.E.M.; Santos, J.A.; Pinheiro, L.V. (2009) - In-situ and Model Wave Characterization at the Alfeite Beach. Journal of Coastal Research, ISSN 07490258. SI56(ICS2009 Proceedings):168-172. Disponível em http://e-geo.fcsh.unl.pt/ICS2009/_docs/ICS2009_ Volume_I/168.172_R.Capitao_ICS2009.pdf.

Carvalho, M.M. (1989) - Sea wave simulation. In: R. Martins (ed.), Recent Advances in Hydraulic Physical Modelling, pp. 447-502, Kluwer Academic Publishers, Dordrecht, Holanda. ISBN: 0-7923-0196-X.

Goda, Y. (1985) - Random Seas and Design of Maritime Structures. 444p. University of Tokyo Press, Toquio, Japão. ISBN: 981-02-3256-X.

GODAE (2010) - Global Ocean Data Assimilation Experiment, U.S. Navy, Monterey, CA, USA. Disponível em http://www.usgodae.org/.

Guilherme, L.; Santos, J.A.; Fortes, C.J.E.M.; Simões,
A. (2009) - Validação da metodologia utilizada para a previsão da agitação marítima implementada no projecto MOIA. Congreso de Métodos Numéricos en Ingeniería 2009, pp 435 (CDRom), Barcelona, Spain. ISBN: 97884-96736-66-5.

Hasselmann, K.; Barnett, T.P.; Bouws, E.; Carlson, H.; Cartwright, D.E.; Enke, K. ; Ewing, J.A. ; Gienapp, H. ; Hasselmann, D.E. ; Kruseman, P. ; Meerburg, A. ; Mller, P.; Olbers, D.J. ; Richter, K. ; Sell, W. ; Walden, H. (1973) - Measurements of Wind-Wave Growth and Swell Decay During the Joing NorthSea Wave Project (JONSWAP), Deutsche Hydrographische Zeitschrift, A8(12).

Lee, D.; Wang, H. (1984) - Measurement of surface waves from subsurface gauge. In: B.L. Edge (ed.), Coastal Engineering 1984: Proceedings of the 19th International Conference on Coastal Engineering, pp. 271-286, ASCE, New York, NY, U.S.A.. ISBN: 9780872624382.

Pires-Silva, A.A., Makararynskyy, O.; Monbaliu, J.; Ventura-Soares, C.; Coelho, E. (2002) - WAM/SWAN Simulations in an Open Coast: Comparisons with ADCP Measurements. Proc. of the 6th Int. Symposium Littoral 2002, pp.169-173, Porto, Portugal.

Rusu, L., Pilar, P.; Guedes Soares, C. (2005a) - Reanalysis of the wave conditions in the approaches to the Portuguese port of Sines, In: C. Guedes Soares, Y. Garbatov \& N Fonseca (eds.), Maritime Transportation and Exploitation of Ocean and Coastal Resources, Vol. 2, pp. 1137-1142, Francis \& Taylor Group, Lisboa, Portugal.

Rusu, L., Pilar, P.; Guedes Soares, C. (2005b) - Hindcasts of the Wave Conditions in Approaches to Ports of the North of Portugal, Proceedings Fifth International Symposium on Ocean Wave Measurement and Analysis (WAVES 2005), CD edition, 9p., Madrid, Espanha.

Rusu, L., Pilar, P.; Guedes Soares, C. (2008a) - Evaluation of the wave conditions in Madeira Archipelago with spectral models. Ocean Engineering, 35(13):1357-1371. DOI:10.1016/j.oceaneng.2008.05.007.

Rusu, L., Pilar, P.; Guedes Soares, C. (2008b) - Hindcast of the wave conditions along the west Iberian coast. Coastal Engineering, 55(11):906-919. DOI:10.1016/j.coastaleng.2008.02.029.

Rusu, L., Pilar, P.; Guedes Soares, C. (2009) - Influence of wind resolution on the prediction of waves generated in a estuary. Journal of Coastal Research, SI56(ICS2009 Proceedings):1419-1423. Disponível em http://e-geo.fcsh.unl.pt/ICS2009/_docs/ICS2009_ Volume_II/1419.1423_L.Rusu_ICS2009.pdf.

Rusu, L.; Bernardino, M.; Guedes Soares, C. (2011) - Modelling the influence of currents on wave propagation at the entrance of the Tagus estuary, Ocean Engineering, 38(10):1174-1183. DOI:10.1016/j.oceaneng.2011.05.016.

Santos, J.A.; Coli, A.B.; Capitão, R.; Fortes, C.J.E.M. (2007) - Wave Forecast at the Tagus Estuary by Using the SWAN Model. Proceedings of the Seventeenth (2007) International Offshore and Polar Engineering Conference. ISOPE, 2007. pp. 2348-2355. Cupertino, CA, U.S.A. ISBN: 978-1-880653-68-5.

Santos, J.A.; Guilherme, L.; Fortes, C.J.E.M.; Pinheiro, L.V.; Simões, A. (2009) - Coupling Numerical Models 
for Wave Propagation in the MOIA Package. Journal of Coastal Research, SI56(ICS2009 Proceedings):544-548. Disponível em http://e-geo.fcsh.unl.pt/ICS2009/_docs/ ICS2009_Volume_I/544.548_J.A.Santos_ICS2009.pdf. Silva, F.; Pinto, J.P.; Almeida, S. (2009) - Operational wave forecast system for the Portuguese Coast. Journal of Coastal Research. SI56(ICS2009 Proceedings):10551059, Disponível em http://e-geo.fcsh.unl.pt/ICS2009/_ docs/ICS2009_Volume_II/1055.1059__F.Sansana Silva_ ICS2009.pdf.

Teles, M.J.; Pires-Silva, A.A.; Belo-Pereira, M.; Fortes, C.J. (2009) - O Modelo SWAN em Regime Não Estacionário: Sensibilidade à Resolução do Campo de Ventos e às
Condiçóes Fronteira de Mar, Actas das $6^{\text {as }}$ Jornadas Portuguesas de Engenharia Costeira e Portuária, CDROM, Funchal, Madeira, Portugal.

Tolman, H. L. (1999) - User manual and system documentation of WAVEWATCH-III version 1.18, 110p., N.O.A.A., National Centers for Environmental Prediction. OMB Technical Note 166. Camp Springs, MD, U.S.A.

Tolman, H. L. (2002) - User manual and system documentation of WAVEWATCH-III version 2.22. 133p., Technical Note, N.O.A.A., National Centers for Environmental Prediction. OMB Technical Note 222. Camp Springs, MD, U.S.A. 